\title{
COMPLETE CMC HYPERSURFACES IN THE HYPERBOLIC SPACE WITH PRESCRIBED GAUSS MAPPING
}

\author{
ABDÊNAGO BARROS, CÍCERO AQUINO, AND HENRIQUE DE LIMA
}

(Communicated by Lei $\mathrm{Ni}$ )

\begin{abstract}
Our aim in this paper is to show that a complete hypersurface $x: M^{n} \rightarrow \mathbb{H}^{n+1}$ immersed with constant mean curvature into the hyperbolic space $\mathbb{H}^{n+1}$ is totally umbilical provided that its Gauss mapping $\nu$ has some suitable behavior. In this setting, our first result requires that the image $\nu(M)$ lies in a totally umbilical spacelike hypersurface of the de Sitter space $\mathbb{S}_{1}^{n+1}$, while in our second one we suppose that $M^{n}$ has scalar curvature bounded from below and that $\nu(M)$ is contained in the closure of a domain enclosed by a totally umbilical spacelike hypersurface of $\mathbb{S}_{1}^{n+1}$ determined by some vector $a$ of the Minkowski space $\mathbb{L}^{n+2}$, with the tangential component of $a$ with respect to $M^{n}$ having Lebesgue integrable norm.
\end{abstract}

\section{InTRODUCTION AND STATEMENTS OF THE RESUlts}

Let $x: M^{n} \rightarrow \mathbb{Q}^{n+1}$ be an immersion of an orientable Riemannian manifold $M^{n}$ in a space form $\mathbb{Q}^{n+1}$ and $\nu: M^{n} \rightarrow \mathbb{Q}^{n+1}$ its associated Gauss mapping. When $\mathbb{Q}^{n+1}$ is a Euclidean space and $x$ is a complete graph of a smooth function $f: \mathbb{R}^{n} \rightarrow \mathbb{R}$, its Gauss mapping is contained in an open hemisphere of $\mathbb{S}^{n}$. The behavior of the Gauss mapping gives a deeper consequence for the immersion. For instance, one of the most celebrated theorems of the theory of minimal surfaces in $\mathbb{R}^{3}$ is Bernstein's theorem [4, which establishes that the only complete minimal graphs in $\mathbb{R}^{3}$ are the planes. This result was extended under the weaker hypothesis that the image of the Gauss mapping of $M^{2}$ lies in an open hemisphere of $\mathbb{S}^{2}$, as we can see in the work of Barbosa and do Carmo [3]. Independently, de Giorgi [5] and Simons 14] showed that a compact minimal hypersurface $M^{n}$ of the Euclidean sphere $\mathbb{S}^{n+1}$ must be a totally geodesic sphere provided that the image of its Gauss mapping lies in an open hemisphere of $\mathbb{S}^{n+1}$. Such a result was extended by Nomizu and Smyth in 9] to constant mean curvature hypersurfaces of $\mathbb{S}^{n}$, proving that a compact connected orientable manifold $M^{n}$ of dimension $n \geq 2$ immersed in the sphere $\mathbb{S}^{n+1}$ with constant mean curvature is a hypersphere if the Gauss image of $M^{n}$ lies in a closed hemisphere of $\mathbb{S}^{n+1}$.

More recently, using the Lorentz model of the hyperbolic space $\mathbb{H}^{n+1}$ (for details, see Section 2), the second and third authors 2] showed that a constant mean curvature complete hypersurface $M^{n}$ which is contained in a geodesic ball of $\mathbb{H}^{n+1}$ and such that the image of the Gauss mapping lies in a totally umbilical spacelike

Received by the editors August 6, 2012 and, in revised form, October 10, 2012.

2010 Mathematics Subject Classification. Primary 53C42; Secondary 53C50.

Key words and phrases. Hyperbolic space, complete hypersurfaces, spacelike hypersurfaces, totally umbilical hypersurfaces, mean curvature, Gauss mapping. 
hypersurface of the de Sitter space $\mathbb{S}_{1}^{n+1}$ must be a totally umbilical geodesic sphere of $\mathbb{H}^{n+1}$. Moreover, in the case that $M^{n}$ is contained between two horospheres (hyperspheres) of $\mathbb{H}^{n+1}$ determined by some nonzero null (spacelike) vector $a$ of the Minkowski space $\mathbb{L}^{n+2}$ and with the image of its Gauss mapping contained in a totally umbilical spacelike hypersurface of $\mathbb{S}_{1}^{n+1}$ determined by $a$, they proved that $M^{n}$ must be a horosphere (hypersphere) of $\mathbb{H}^{n+1}$.

Here, motivated by the works previously described, we apply a suitable Simonstype formula due to Nomizu and Smyth [10] jointly with the well known generalized maximum principle of Omori-Yau [11,15] in order to obtain the following extension of the results of [2] mentioned above.

Theorem 1.1. The only complete constant mean curvature hypersurfaces immersed in $\mathbb{H}^{n+1}$ such that the image of the Gauss mapping lies in a totally umbilical spacelike hypersurface of $\mathbb{S}_{1}^{n+1}$ are the totally umbilical ones.

In [8], Montiel proved that if a complete spacelike hypersurface $\Sigma^{n}$ in the de Sitter space $\mathbb{S}_{1}^{n+1}$ with constant mean curvature $H \geq 1$ is such that the image of its Gauss mapping is contained in the closure of the interior domain enclosed by a horosphere of $\mathbb{H}^{n+1}$, then its mean curvature is, in fact, equal to 1 . When $n=2$, this implies that $\Sigma^{2}$ is also a totally umbilical surface and, hence, the image of its Gauss mapping is exactly a horosphere of $\mathbb{H}^{3}$. In our second rigidity theorem, we establish a sort of dual for the result of Montiel mentioned above. For this, we use as our main analytical tool an extension of the classical Hopf theorem on a complete noncompact Riemannian manifold due to Yau [16] (cf. Lemma 3.2).

In what follows, we denote by $a^{\top}$ the tangential component of a vector $a \in$ $\mathbb{L}^{n+2}$ with respect to an immersion $x: M^{n} \rightarrow \mathbb{H}^{n+1} \subset \mathbb{L}^{n+2}$ and, according to the terminology established in [8], we say that the image of its Gauss mapping $\nu$ is contained in the closure of a domain enclosed by a totally umbilical spacelike hypersurface of $\mathbb{S}_{1}^{n+1}$ determined by some vector $a \in \mathbb{L}^{n+2}$ when the angle function $\langle\nu, a\rangle$ does not change sign on $M^{n}$.

Theorem 1.2. The only complete constant mean curvature hypersurfaces immersed in $\mathbb{H}^{n+1}$ with scalar curvature bounded from below and whose image of the Gauss mapping is contained in the closure of a domain enclosed by a totally umbilical spacelike hypersurface of $\mathbb{S}_{1}^{n+1}$ determined by some vector $a \in \mathbb{L}^{n+2}$, with a ${ }^{\top}$ having Lebesgue integrable norm along them, are the totally umbilical ones.

The proofs of Theorems 1.1 and 1.2 are given in Section 3 .

\section{Preliminaries}

Throughout this paper we consider the Lorentz model of the hyperbolic space $\mathbb{H}^{n+1}$ obtained by furnishing the hyperquadric $\left\{p \in \mathbb{L}^{n+2} ;\langle p, p\rangle=-1, p_{n+2}>0\right\}$ with the Riemannian metric induced by the Lorentz metric of the Minkowski space $\mathbb{L}^{n+2}$. In this setting, let $x: M^{n} \rightarrow \mathbb{H}^{n+1} \subset \mathbb{L}^{n+2}$ be a connected orientable hypersurface immersed into $\mathbb{H}^{n+1}$. We recall that a unit normal globally defined vector field $\nu$ of $M^{n}$ can be regarded as a mapping $\nu: M^{n} \rightarrow \mathbb{S}_{1}^{n+1}$, where $\mathbb{S}_{1}^{n+1}$ denotes the $(n+1)$-dimensional unitary de Sitter space; that is,

$$
\mathbb{S}_{1}^{n+1}=\left\{p \in \mathbb{L}^{n+2} ;\langle p, p\rangle=1\right\} .
$$


In order to set up the notation, we will denote by $\nabla^{0}, \bar{\nabla}$ and $\nabla$ the Levi-Civita connections of $\mathbb{L}^{n+2}, \mathbb{H}^{n+1}$ and $M^{n}$, respectively. Then the Gauss and Weingarten formulas for $M^{n}$ in $\mathbb{H}^{n+1}$ are given, respectively, by

$$
\nabla^{0}{ }_{X} Y=\nabla_{X} Y+\langle A X, Y\rangle \nu+\langle X, Y\rangle x
$$

and

$$
A X=-\bar{\nabla}_{X} \nu=-\nabla_{X}^{0} \nu
$$

for all tangent vector fields $X, Y \in \mathfrak{X}(M)$, where $A$ stands for the shape operator of $M^{n}$ with respect to $\nu$.

In what follows, for a fixed arbitrary vector $a \in \mathbb{L}^{n+2}$, let us consider the height and the angle functions, defined respectively by $l_{a}=\langle x, a\rangle$ and $f_{a}=\langle\nu, a\rangle$. A direct computation allows us to conclude that the gradient of such functions are given by $\nabla l_{a}=a^{\top}$ and $\nabla f_{a}=-A\left(a^{\top}\right)$, where $a^{\top}$ is the orthogonal projection of $a$ onto the tangent bundle $T M$, which is given by

$$
a^{\top}=a-f_{a} \nu+l_{a} x .
$$

Taking into account that $\nabla^{0} a=0$ and using the Gauss and Weingarten formulas, we have

$$
\nabla_{X} a^{\top}=f_{a} A X+l_{a} X
$$

for all $X \in \mathfrak{X}(M)$. We now use (2.1) and the Codazzi equation to deduce

$$
\nabla_{X} A\left(a^{\top}\right)=f_{a} A^{2} X+l_{a} A X+\left(\nabla_{a^{\top}} A\right)(X)
$$

for all $X \in \mathfrak{X}(M)$. Thus, according to [12] (see also [1]), it follows from the last two identities that

$$
\Delta l_{a}=n H f_{a}+n l_{a}
$$

and

$$
\Delta f_{a}=-|A|^{2} f_{a}-n H l_{a}-n\left\langle\nabla H, a^{\top}\right\rangle,
$$

where $H=(1 / n) \operatorname{trace}(A)$ is the mean curvature of $M^{n}$.

For what follows, it is also convenient to consider the traceless operator associated to the second fundamental form, $\Phi: \mathfrak{X}(M) \rightarrow \mathfrak{X}(M)$, which is given by

$$
\Phi(X)=A X-H X,
$$

for all $X \in \mathfrak{X}(M)$. It is easily checked that the Hilbert-Schmidt norm of $\Phi$ (that is, $|\Phi|^{2}=\operatorname{tr}\left(\Phi^{*} \Phi\right)$, where $\Phi^{*}$ stands for the adjoint of $\left.\Phi\right)$ satisfies

$$
|\Phi|^{2}=|A|^{2}-n H^{2} .
$$

Consequently, we have that $|\Phi|^{2}=0$ if and only if $M^{n}$ is totally umbilical.

Now, we will recall the description of the totally umbilical spacelike hypersurfaces of $\mathbb{S}_{1}^{n+1}$ due to Montiel in [7]. Let $\mathcal{L}_{\rho}$ be the spacelike hypersurface immersed into $\mathbb{S}_{1}^{n+1}$ given by

$$
\mathcal{L}_{\rho}=\left\{p \in \mathbb{S}_{1}^{n+1} ;\langle p, a\rangle=\rho\right\},
$$

where $a \in \mathbb{L}^{n+2},\langle a, a\rangle=1,0,-1$ and $\rho^{2}>\langle a, a\rangle$. Then, for $p \in \mathcal{L}_{\rho}$,

$$
\nu(p)=\frac{1}{\sqrt{\rho^{2}-\langle a, a\rangle}}(a-\rho p) \in \mathbb{H}^{n+1}
$$


is a unit normal vector field for $\mathcal{L}_{\rho}$. Consequently, the shape operator $A$ of $\mathcal{L}_{\rho}$ is given by

$$
A X=\frac{\rho}{\sqrt{\rho^{2}-\langle a, a\rangle}} X
$$

for all smooth vector fields $X$ tangent to $\mathcal{L}_{\rho}$ (cf. [7, Example 1). Hence, $\mathcal{L}_{\rho}$ is totally umbilical with constant mean curvature $H=\frac{\rho}{\sqrt{\rho^{2}-\langle a, a\rangle}}$. In fact, it can be verified that:

(1) if $a$ is a unit spacelike vector, then $\mathcal{L}_{\rho}$ is isometric to an $n$-dimensional hyperbolic space of constant sectional curvature $-\frac{1}{\rho^{2}-1}$ and $H^{2}>1$;

(2) if $a$ is a nonzero null vector, then $\mathcal{L}_{\rho}$ is isometric to a Euclidean space $\mathbb{R}^{n}$ and $H^{2}=1$;

(3) if $a$ is a unit timelike vector, then $\mathcal{L}_{\rho}$ is isometric to an $n$-dimensional sphere of constant sectional curvature $\frac{1}{\rho^{2}+1}$ and $0 \leq H^{2}<1$.

To close this section, we quote a suitable characterization of totally umbilical hypersurfaces in a semi-Riemannian space form due to Kim et al. [6], which corresponds to a converse for a theorem due to Sharma and Duggal in [13].

Lemma 2.1. Let $M^{n}$ be a connected semi-Riemannian hypersurface of a semiRiemannian space form $\bar{M}^{n+1}(c)$. Suppose that $\bar{M}^{n+1}(c)$ carries a conformal vector field $V$ whose tangential component $V^{\top}$ on $M^{n}$ becomes a conformal vector field. Then, one of the following holds:

(i) $M^{n}$ is a totally umbilical hypersurface;

(ii) the restriction of $V$ to $M^{n}$ reduces to a tangent vector field on $M^{n}$.

\section{Proofs of Theorems 1.1 and 1.2}

In order to prove our first result, we quote the well known generalized maximum principle due to Omori-Yau [11] and Yau [15.

Lemma 3.1. Let $M^{n}$ be an $n$-dimensional complete Riemannian manifold whose Ricci curvature is bounded from below and let $u: M^{n} \rightarrow \mathbb{R}$ be a smooth function which is bounded from above on $M^{n}$. Then there exists a sequence $\left(p_{k}\right)_{k \geq 1}$ in $M^{n}$ such that

$$
\lim _{k} u\left(p_{k}\right)=\sup _{M} u, \quad \lim _{k}|\nabla u|\left(p_{k}\right)=0 \quad \text { and } \lim _{k} \sup \Delta u\left(p_{k}\right) \leq 0 .
$$

Proof of Theorem 1.1. Let $x: M^{n} \rightarrow \mathbb{H}^{n+1}$ be a complete immersed hypersurface with constant mean curvature $H$ and let us denote by $A$ its Weingarten operator with respect to a globally defined normal vector field $\nu$. Using the characterization of totally umbilical spacelike hypersurfaces of $\mathbb{S}_{1}^{n+1}$ described in Section 2, we have from our hypothesis under the image of the Gauss mapping $\nu$ of $M^{n}$ that there exists a vector $a \in \mathbb{L}^{n+2}$ and a real number $\tau$ such that the angle function $f_{a}$ of $M^{n}$ satisfies $f_{a}=\langle\nu, a\rangle=\tau$ on $M^{n}$, with $\tau^{2}>\langle a, a\rangle$.

If $\tau=0$, then it immediately follows from (2.1) that the Hessian of the height function $l_{a}=\langle x, a\rangle$ satisfies $\nabla^{2} l_{a}=l_{a} g$, where $g$ stands for the Riemannian metric of $M^{n}$. Consequently, we conclude that $\nabla l_{a}=a^{\top}$ is a conformal vector field on $M^{n}$. From Lemma 2.1, since $H=0$ in this case, we obtain that $M^{n}$ is a totally geodesic hypersurface of $\mathbb{H}^{n+1}$. 
If $\tau \neq 0$, then $H \neq 0$ and formula (2.3) gives

$$
|A|^{2}=-\frac{n H}{\tau} l_{a}
$$

Now we use (3.1) to conclude that

$$
\frac{\tau}{H} l_{a}=-\frac{\tau^{2}}{n H^{2}}|\Phi|^{2}-\tau^{2},
$$

where $\Phi=A-H I$ was previously defined. Therefore, the height function $l_{a}$ satisfies $\left|l_{a}\right| \geq \beta$ for some positive constant $\beta$. We can assume, without loss of generality, that $l_{a}$ is a strictly positive function on $M^{n}$.

We claim that the height function $l_{a}$ is upper bounded. Indeed, since the mean curvature $H$ of $M^{n}$ is constant and $a^{\top}=\nabla l_{a}$, we obtain from (3.1) the following equality:

$$
a^{\top}\left(|A|^{2}\right)=-\frac{n H}{\tau}\left|\nabla l_{a}\right|^{2} .
$$

Now we choose a local orthonormal frame $\left\{e_{1}, \ldots, e_{n}\right\}$ on a neighborhood $\mathcal{U} \subset M^{n}$ which is geodesic at a point $p \in \mathcal{U}$. Thus, since $A$ is a symmetric operator and $A\left(a^{\top}\right)=0$, the Codazzi equation gives

$$
a^{\top}\left(|A|^{2}\right)=2 \sum_{i=1}^{n}\left\langle\nabla_{a^{\top}} A e_{i}, A e_{i}\right\rangle=-2 \sum_{i=1}^{n}\left\langle A^{2}\left(\nabla_{e_{i}} a^{\top}\right), e_{i}\right\rangle .
$$

On the other hand, since $\nabla_{e_{i}} a^{\top}=\tau A e_{i}+l_{a} e_{i}$ at $p$, we compare (3.1), (3.2) and (3.3) to deduce

$$
n H \operatorname{tr}\left(A^{3}\right)=|A|^{4}+\frac{n^{2} H^{2}}{2 \tau^{2}}\left|\nabla l_{a}\right|^{2} .
$$

In [10], Nomizu and Smyth obtained the following Simons-type formula:

$$
\frac{1}{2} \Delta|A|^{2}=-n|A|^{2}-|A|^{4}+n^{2} H^{2}+n H \operatorname{tr}\left(A^{3}\right)+|\nabla A|^{2} .
$$

Returning to our context, since $H$ is constant and $f_{a}=\tau$, it follows from (2.2) and (3.1) that $\Delta|A|^{2}=\Delta|\Phi|^{2}=n|\Phi|^{2}$. Therefore, we can use (3.4) and (3.5) to deduce

$$
|\Phi|^{2}=\frac{n H^{2}}{3 \tau^{2}}\left|\nabla l_{a}\right|^{2}+\frac{1}{3 n}|\nabla A|^{2} .
$$

Using that $l_{a}$ is a strictly positive function and the identity $\left|\nabla l_{a}\right|^{2}+\tau^{2}-l_{a}^{2}=\langle a, a\rangle$, we obtain from (3.6) the following expression:

$$
\left(\frac{H^{2}}{3 \tau^{2}}\langle a, a\rangle+\frac{2 H^{2}}{3}\right) \frac{1}{l_{a}}+\frac{H^{2}}{3 \tau^{2}} l_{a} \leq-\frac{H}{\tau} .
$$

Now we are in a position to prove that the height function $l_{a}$ is bounded. Suppose by contradiction that there exists a sequence of points $\left(q_{k}\right)_{k>1}$ in $M^{n}$ such that $l_{a}\left(q_{k}\right) \rightarrow+\infty$ when $k \rightarrow \infty$. But, from the above inequality we obtain, after a straightforward computation, that

$$
\lim _{k} l_{a}\left(q_{k}\right) \leq-\frac{3 \tau}{H}
$$

which gives a contradiction. Consequently, $l_{a}$ is bounded and we finish our claim. 
Therefore, we can use (3.1) to conclude that $|A|^{2}$ is also bounded. On the other hand, from the Gauss equation we have that the Ricci curvature tensor of $M^{n}$, denoted by $\operatorname{Ric}_{M}$, is given by

$$
\operatorname{Ric}_{M}(X, Y)=-(n-1)\langle X, Y\rangle+n H\langle A X, Y\rangle-\langle A X, A Y\rangle,
$$

for all $X, Y \in \mathfrak{X}(M)$. Thus, using the Cauchy-Schwarz inequality, from (3.9) we have that

$$
\operatorname{Ric}_{M}(X, X) \geq\left(1-n-n|H||A|-|A|^{2}\right)|X|^{2},
$$

for all $X \in \mathfrak{X}(M)$. Since $|A|^{2}$ is bounded and $H$ is constant, we conclude from (3.10) that $\operatorname{Ric}_{M}$ is bounded from below. So, we can apply Lemma 3.1 to pick out a sequence of points $\left(p_{k}\right)_{k \geq 1}$ in $M^{n}$ such that

$$
\lim _{k}|\Phi|^{2}\left(p_{k}\right)=\sup _{M}|\Phi|^{2} \text { and } \lim _{k} \sup \Delta|\Phi|^{2}\left(p_{k}\right) \leq 0 .
$$

Since $\Delta|\Phi|^{2}=n|\Phi|^{2}$, we get

$$
0 \geq \lim _{k} \sup \Delta|\Phi|^{2}\left(p_{k}\right)=n \sup _{M}|\Phi|^{2} \geq 0 .
$$

Hence, $\sup _{M}|\Phi|^{2}=0$ and, therefore, $|\Phi|^{2}=0$ on $M^{n}$, which means that $M^{n}$ is a totally umbilical hypersurface of $\mathbb{H}^{n+1}$ which was to be proved.

Before we present the proof of our second result, we will quote an extension of the classical Hopf maximum principle for an $n$-dimensional complete Riemannian manifold $M^{n}$ due to Yau [16. In what follows, $\mathcal{L}^{1}(M)$ stands for the space of Lebesgue integrable functions on $M^{n}$.

Lemma 3.2. Let $M^{n}$ be an $n$-dimensional complete Riemannian manifold and let $u: M^{n} \rightarrow \mathbb{R}$ be a smooth function. If $u$ is a subharmonic (or superharmonic) function with $|\nabla u| \in \mathcal{L}^{1}(M)$, then $u$ must actually be harmonic.

Proof of Theorem 1.2. Let $x: M^{n} \rightarrow \mathbb{H}^{n+1}$ be a complete immersed hypersurface with constant mean curvature $H$. Initially, we observe that our hypothesis under the image of the Gauss mapping $\nu$ of $M^{n}$ amounts to the fact that, for some vector $a \in \mathbb{L}^{n+2}$, the angle function $f_{a}=\langle\nu, a\rangle$ does not change sign on $M^{n}$.

Now a straightforward computation allows us to conclude that the Hessian of the height function $l_{a}=\langle x, a\rangle$ satisfies

$$
\left|\nabla^{2} l_{a}\right|^{2}=|\Phi|^{2} f_{a}^{2}+\frac{1}{n}\left(\Delta l_{a}\right)^{2}
$$

where $A$ is the Weingarten operator of $M^{n}$ while $\Phi$ is its associated traceless operator.

Moreover, since the mean curvature of $M^{n}$ is constant, we have from formulas (2.2) and (2.3) that

$$
\Delta\left(f_{a}+H l_{a}\right)=-|\Phi|^{2} f_{a} .
$$

Thus, $\Delta\left(f_{a}+H l_{a}\right)$ does not change sign on $M^{n}$.

On the other hand, from (3.9) we have that the scalar curvature $R$ of $M^{n}$ satisfies

$$
R=n(1-n)+n^{2} H^{2}-|A|^{2} .
$$

Since $R$ is bounded from below, we get that $|A|^{2}$ is bounded on $M^{n}$. Consequently,

$$
\left|\nabla\left(f_{a}+H l_{a}\right)\right|=\left|-A\left(a^{\top}\right)+H a^{\top}\right| \leq(|A|+|H|)\left|a^{\top}\right| \in \mathcal{L}^{1}(M) .
$$


Thus, from Lemma 3.2 we conclude that the function $f_{a}+H l_{a}$ is harmonic and, hence, $|\Phi|^{2} f_{a}=0$ on $M^{n}$. On the other hand, since the Hilbert-Schmidt norm of $\nabla^{2} l_{a}-\frac{1}{n} \Delta l_{a} g$ satisfies $\left|\nabla^{2} l_{a}-\frac{1}{n} \Delta l_{a} g\right|^{2}=\left|\nabla^{2} l_{a}\right|^{2}-\frac{1}{n}\left(\Delta l_{a}\right)^{2}$, we use (3.11) to conclude that

$$
\nabla^{2} l_{a}=\frac{1}{n}\left(\Delta l_{a}\right) g
$$

where $g$ stands for the induced metric of $M^{n}$. Therefore, $\nabla l_{a}=a^{\top}$ is a conformal vector field on $M^{n}$ and, since $a$ cannot be a tangent vector to the hypersurface, we have from Lemma 2.1 that $M^{n}$ is a totally umbilical hypersurface of $\mathbb{H}^{n+1}$. This completes the proof of our theorem.

\section{ACKNOWLEDGMENTS}

The first author was partially supported by $\mathrm{CNPq}$, Brazil, and the third author was partially supported by CNPq, Brazil, grant 300769/2012-1. The authors would like to thank the referee for giving some valuable suggestions which improved the paper.

\section{RefERENCES}

[1] Luis J. Alías, On the stability index of minimal and constant mean curvature hypersurfaces in spheres, Rev. Un. Mat. Argentina 47 (2006), no. 2, 39-61 (2007). MR2301375 (2008c:53057)

[2] C. P. Aquino and H. F. de Lima, On the Gauss map of complete CMC hypersurfaces in the hyperbolic space, J. Math. Anal. Appl. 386 (2012), no. 2, 862-869, DOI 10.1016/j.jmaa.2011.08.046. MR2834793(2012k:53106)

[3] J. L. Barbosa and M. do Carmo, Stable minimal surfaces, Bull. Amer. Math. Soc. 80 (1974), 581-583. MR0336533(49 \#1307)

[4] Serge Bernstein, Sur les surfaces définies au moyen de leur courbure moyenne ou totale (French), Ann. Sci. École Norm. Sup. (3) 27 (1910), 233-256. MR.1509123

[5] Ennio De Giorgi, Una estensione del teorema di Bernstein (Italian), Ann. Scuola Norm. Sup. Pisa (3) 19 (1965), 79-85. MR0178385 (31 \#2643)

[6] Dong-Soo Kim, Seon-Bu Kim, Young Ho Kim, and Seong-Hee Park, Conformal vector fields and totally umbilic hypersurfaces, Bull. Korean Math. Soc. 39 (2002), no. 4, 671-680, DOI 10.4134/BKMS.2002.39.4.671. MR1939565(2003h:53077)

[7] Sebastián Montiel, An integral inequality for compact spacelike hypersurfaces in de Sitter space and applications to the case of constant mean curvature, Indiana Univ. Math. J. 37 (1988), no. 4, 909-917, DOI 10.1512/iumj.1988.37.37045. MR982837(90c:53167)

[8] Sebastián Montiel, Complete non-compact spacelike hypersurfaces of constant mean curvature in de Sitter spaces, J. Math. Soc. Japan 55 (2003), no. 4, 915-938, DOI 10.2969/jmsj/1191418756. MR2003752 (2004h:53095)

[9] Katsumi Nomizu and Brian Smyth, On the Gauss mapping for hypersurfaces of constant mean curvature in the sphere, Comment. Math. Helv. 44 (1969), 484-490. MR0257939 (41 \#2588)

[10] Katsumi Nomizu and Brian Smyth, A formula of Simons' type and hypersurfaces with constant mean curvature, J. Differential Geometry 3 (1969), 367-377. MR0266109 (42 \#1018)

[11] Hideki Omori, Isometric immersions of Riemannian manifolds, J. Math. Soc. Japan 19 (1967), 205-214. MR0215259(35 \#6101)

[12] Harold Rosenberg, Hypersurfaces of constant curvature in space forms, Bull. Sci. Math. 117 (1993), no. 2, 211-239. MR.1216008 (94b:53097)

[13] R. Sharma and K. L. Duggal, Characterization of an affine conformal vector field, C. R. Math. Rep. Acad. Sci. Canada 7 (1985), no. 3, 201-205. MR789313 (86j:53065)

[14] James Simons, Minimal varieties in riemannian manifolds, Ann. of Math. (2) 88 (1968), 62-105. MR0233295 (38 \#1617) 
[15] Shing Tung Yau, Harmonic functions on complete Riemannian manifolds, Comm. Pure Appl. Math. 28 (1975), 201-228. MR0431040 (55 \#4042)

[16] Shing Tung Yau, Some function-theoretic properties of complete Riemannian manifold and their applications to geometry, Indiana Univ. Math. J. 25 (1976), no. 7, 659-670. MR0417452 (54 \#5502)

Departamento de Matemática, Universidade Federal do Ceará, 60455-760 Fortaleza, CEArÁ, Brazil

E-mail address: abbarros@mat.ufc.br

Departamento de Matemática, Universidade Federal do Piauí, 64049-550 Teresina, PiAuí, BRAZIL

E-mail address: cicero.aquino@ufpi.edu.br

Departamento de Matemática e Estatística, Universidade Federal de Campina Grande, 58109-970 Campina Grande, Paraíba, Brazil

E-mail address: henrique@dme.ufcg.edu.br 